\title{
Deserción y retención universitaria: una discusión bibliográfica
}

\author{
María del Rosario Fernández-Hileman*, Ángela Corengia ${ }^{\star *}$, Julio Durand ${ }^{\star * *}$
}

* Doctoranda en Educación. Profesora ayudante de la Escuela de Educación de la Universidad Austral, Campus Pilar, Buenos Aires, Argentina.

Correo electrónico:

mrfernandez@austral.edu.ar

** Doctora en Educación. Profesora asociada de la Escuela de Educación de la Universidad Austral, Campus Pilar, Buenos Aires, Argentina.

Correo electrónico:

acorengia@asutral.edu.ar

*** Doctor en Educación. Profesor titular y Director de la Escuela de Educación de la Universidad Austral, Campus Pilar, Buenos Aires, Argentina.

Correo electrónico:

jdurand@austral.edu.ar

Recibido: 7 de noviembre del 2013

Aprobado: 13 de julio del 2014

Cómo citar este artículo: FernándezHileman, M. R., Corengia, A. y Durand, D. (2014). Deserción y retención universitaria: una discusión bibliográfica. Pensando Psicología, 10(17), 85-96. doi: http://dx. doi. org/10.16925/pe.v10i17.787

\begin{abstract}
Resumen
El objetivo de este artículo es presentar una discusión bibliográfica acerca del fenómeno de la deserción y retención en la universidad. Para ello, se realizó una revisión y síntesis de los principales estudios empíricos y perspectivas teóricas que abordan esta temática en Argentina y otros países. Para comenzar, se muestra el problema de la deserción en números, algunas precisiones terminológicas y su contextualización nacional (Argentina) e internacional. Posteriormente se desarrollan tanto las perspectivas conceptuales sustantivas basadas en teorías sociológicas, psicológicas, organizacionales, económicas y culturales, como los estudios empíricos que sustentan estas y otras precisiones teóricas. En el marco de estos estudios empíricos, también se relevan acciones y programas llevados a cabo a lo largo de los últimos cuarenta años para promover la retención en la educación superior. Finalmente, se exponen las conclusiones a modo de insumo para futuras investigaciones.
\end{abstract}

Palabras clave: educación superior, deserción, retención, revisión bibliográfica.

\section{University Desertion and Retention: A Bibliographical Study}

\section{Abstract}

This article presents a bibliographical study of the phenomena of university desertion and retention, including a review and summary of the main empirical studies and theoretical perspectives that address this subject in Argentina and other countries. Firstly, the problem of desertion is shown in numbers, in specific terms and in Argentinian and international contexts. Next, the article looks at substantive conceptual perspectives based on sociological, psychological, organizational, economic and cultural theories, as well as the empirical studies that support these along with other theoretical clarifications. Within the framework of these empirical studies, the study then highlights actions and programs carried out over the past 40 years to improve retention in higher education. Finally, conclusions are offered as material for future research.

Keywords: higher education, desertion, retention, bibliographical review.

\section{Deserção e retenção universitária: uma discussão bibliográfica}

\section{Resumo}

O objetivo deste artigo é apresentar uma discussão bibliográfica sobre o fenômeno da deserção e retenção na universidade. Para isso, realizaram-se uma revisão e uma síntese dos principais estudos empíricos e perspectivas teóricas que abordam essa temática na Argentina e outros países. Para começar, mostram-se o problema da deserção em números, algumas posições terminológicas e sua contextualização nacional (Argentina) e internacional. Em seguida, desenvolvem-se tanto as perspectivas conceituais substanciais baseadas em teorias sociológicas, psicológicas, organizacionais, econômicas e culturais, quanto os estudos empíricos que sustentam essas e outras precisões teóricas. No marco desses estudos empíricos, também se relevam ações e programas realizados ao longo dos últimos 40 anos para promover a retenção na educação superior. Finalmente, expõem-se as conclusões a modo de insumo para futuras pesquisas.

Palavras-chave: educação superior, deserção, retenção, revisão bibliográfica. 


\section{Introducción}

El panorama mundial de la deserción en la educación superior en las últimas décadas, muestra que Francia y Austria tienen tasas de deserción de un 30\% y 50\%, respectivamente; en Alemania las cifras oscilan entre el $20 \%$ y el 25\%; en Finlandia es del 10\% y en los Países Bajos, entre el 20\% y el 30\%. En el caso de los Estados Unidos existen grandes diferencias entre las tasas de graduación de universidades de gran prestigio como Harvard y Princeton que gradúan aproximadamente al $90 \%$ de sus estudiantes, y otras de carácter público que gradúan menos del $30 \%$ de su población estudiantil. En el caso de Latinoamérica y el Caribe, ${ }^{1}$ para el 2003 la educación superior presentó una cobertura del $28,7 \%$ y una tasa de deserción promedio de 50\%, siendo Guatemala, Uruguay y Bolivia los países con índices más altos de abandono $82 \%$, 72\% y 73\% respectivamente (PinedaBáez, Pedraza-Ortiz, Baquero, Dussán y Ramírez, 2010).

En la Argentina, sólo 27 de cada 100 estudiantes que empiezan la universidad se gradúan. Mientras que en las universidades públicas sólo terminan 23 de cada 100, en las privadas 40 de 100 logran finalizar la carrera. Si bien la matrícula universitaria creció un $18 \%$ entre el 2002 y el 2010, superando así proporcionalmente a la cantidad de matriculados en Brasil, allí se gradúan 4,3 de cada 100 habitantes, mientras que en la Argentina se gradúan 2,5 por cada 100. Esto indica que a pesar de que se ha extendido bastante el acceso a la universidad, son muy pocos los estudiantes que logran concluir sus estudios. Más aún, la Argentina tiene una de las menores tasas de graduación en relación con la cantidad de ingresantes a nivel mundial, y no sólo regional. Prueba de ello es que en Brasil se gradúan en promedio 50 de cada 100 ingresantes, en Chile 59 y en Francia 67 (Centro de Estudios de la Educación Argentina [CEA], 2012). En Colombia, al igual que en la Argentina, a pesar del aumento en el número de estudiantes que ingresan a las universidades, el desafío sigue siendo su permanencia en las instituciones educativas y su egreso como profesionales. El más reciente reporte presentado por la Ministra de Educación señala que la deserción estudiantil en las instituciones de educación superior (IES) colombianas al 2008 era de 44,9\% (Pineda-Báez et al., 2010).

La Organización de las Naciones Unidas para la Educación, la Ciencia y la Cultura (Unesco) por medio del Instituto Internacional para la Educación Superior en América Latina y el Caribe (Iesalc), realizó en el 2004 una investigación cuyo objetivo fue recoger información por países para dimensionar la repitencia y deserción y especificar los factores más relevantes que incidieron en el abandono de los estudios universitarios.
El objetivo de este artículo es presentar una exhaustiva discusión bibliográfica acerca del fenómeno de la deserción que tan negativamente impacta a las instituciones y los sistemas de educación superior $y$, principalmente, a los estudiantes que la padecen. Para ello se realizó una revisión y síntesis de los principales estudios, en los que se observaron sistemáticamente los objetivos y las preguntas de investigación, el marco teórico, la metodología y los resultados, a partir de los cuales surgen una serie de apreciaciones que se exponen en las siguientes partes de este trabajo. La búsqueda bibliográfica abarcó principalmente tesis de posgrado, libros sobre la temática, e investigaciones halladas en journals internacionales. Esta se realizó a través de base de datos en ciencias sociales, particularmente EBsCo, JSTOR y ERIC. Se acotó por "palabras claves en títulos y abstracts" y por "mayor cantidad de veces citados".

\section{Precisiones terminológicas}

Pese a su gravedad, la deserción todavía ha sido poco estudiada en la Argentina, ya que diversos autores aún no han logrado consenso sobre la real dimensión del problema. Las investigaciones sobre fracaso estudiantil en la universidad Argentina (García de Fanelli y Trombetta, 1996; Solimano, 1997; Beguet, Kohan, Castro-Solano y Renault, 2001; Lorenzano y Ferraro, 2003; Foio y Espínola, 2004; García de Fanelli, 2004; Porto y Di Gresia, 2004; Aparicio, 2005 y 2008; Di Gresia, 2007) presentan la deserción vinculada a factores múltiples y diversos como por ejemplo los fisiológicos, psicobiológicos y clima familiar; la inteligencia, el sexo, el tipo de instituciones y los efectos que produce el nivel sociocultural de las familias en el rendimiento. Otros aspectos asociados son el origen social y algunos desequilibrios psicológicos como la inseguridad, ansiedad, falta de confianza en sí mismo, carencia afectiva, inmadurez, agresividad, inhibición y bajas expectativas. Otras investigaciones sobre fracaso arrojan causas de índole académica como la excesiva duración de las carreras, mala calidad de la enseñanza, falta de profesionalización, sistemas de evaluación y selección, escasa orientación vocacional, alto número de alumnos por profesor, enciclopedismo, bajo porcentaje de profesores a tiempo completo, entre otros. En general, a pesar de realizar aportes interesantes, se trata de estudios reducidos a una dimensión, en especial, dejando de lado una perspectiva contextualizante y holística. Según Bellei (2003), la tendencia en materia de 
retención devela que los sistemas educativos se desprenden cada vez más de su cuota de responsabilidad sobre los resultados y "privatizan" el fracaso atribuyéndolo al alumno.

Además, se suma el problema de la falta de univocidad del término "fracaso", con el cual se hace referencia a diferentes aspectos, tales como: bajo rendimiento, repetición de cursos o asignaturas, abandono de la carrera, abandono del sistema universitario e inadaptación, entre otros. Asimismo, la conceptualización de los términos "deserción" y "retención" tampoco ha sido siempre consistente, dado que a lo largo de los años se han utilizado diferentes palabras para explicar lo mismo. Ejemplo de ello son los términos de mortalidad estudiantil (McNeely, 1937; Gekowski y Schwartz, 1961), deserción universitaria (Summerkill, 1962; Spady, 1970; Tinto, 1975), deserción estudiantil (Sexton, 1965; Panos y Astin, 1967; Pantages y Creedon, 1978; Tinto, 1993), retención (Iffert, 1957; Tinto, 1990; Berger, 2002; Braxton y Mundy, 2002) y persistencia (Berger y Milem, 1999; Berger, 2002). Conceptos como retención y persistencia se han utilizado como sinónimos cuando en verdad sus significados divergen. Mientras que con nivel de retención se hace referencia a la tasa de permanencia de los estudiantes en una institución en particular, la persistencia se refiere al hecho de que un determinado estudiante finalice sus estudios y obtenga su respectivo diploma sin tener en cuenta por cuantas instituciones pasó, es decir, si se cambió de universidad o no. En otras palabras, la persistencia refiere a la permanencia a nivel de sistema de educación superior, mientras que la retención alude a esa permanencia pero en cada institución particular. Otros autores como Panaia (2013) prefieren hablar de abandono estudiantil, en lugar de deserción. Dentro de dicho proceso la autora distingue tres secuencias: una secuencia previa con frustraciones, obstáculos, dificultades e inercias; un acontecimiento, que es la decisión de abandonar, de aparición no premeditada, que funciona como un corte en la trayectoria; y una secuencia posterior que muestra en el tiempo las consecuencias de la decisión tomada configurando una bifurcación en la trayectoria de los estudiantes.

Adicionalmente, en las instituciones se consideran varios factores para clasificar el término deserción vinculados con la temporalidad, el alcance y el mecanismo de abandono. En cuanto a la dimensión temporal, que alude al momento o momentos en que ocurre la desvinculación y a su duración, se encuentra la deserción precoz, la deserción temprana y la tardía. Según Castaño, Gallón, Gómez y Vásquez (2004), la primera se presenta cuando el estudiante ha sido admitido por la universidad pero no se matricula, mientras que la segunda se refiere al abandono durante los cuatro primeros semestres de la carrera. La tercera alude al abandono que se da del quinto semestre en adelante. En cuanto a la duración, se puede discriminar entre una interrupción temporal y un abandono definitivo. En relación con el alcance, la deserción puede ser de la carrera solamente, permaneciendo el estudiante dentro de la misma institución pero en otra carrera; de la institución, cuando se opta por continuar con los estudios en otra universidad; o deserción del sistema educativo universitario, cuando se abandonan los estudios superiores definitivamente. Por último, en relación con los mecanismos de abandono, estos están estrechamente relacionados con la reglamentación institucional. Siguiendo a Castaño et al. (2004), se puede decir que existe un retiro forzoso cuando el estudiante transgrede el reglamento estudiantil o presenta un bajo rendimiento académico, mientras que se habla de un abandono voluntario cuando el retiro se da por decisión propia del estudiante a pesar de cumplir con los requisitos reglamentarios establecidos por la institución.

\section{Contextualización del problema en la Argentina y a nivel internacional}

La deserción universitaria es un fenómeno con serias repercusiones en las instituciones de educación superior, tanto desde el ángulo socioeconómico como psicosocial, que tocan al actor involucrado así como al sistema en su conjunto. Los estudios realizados en la Argentina pueden ser clasificados en dos grupos: los que responden a una mirada más socioeconómica del fenómeno, como los trabajos de García de Fanelli y Trombetta (1996); Solimano (1997); Beguet et al. (2001); Lorenzano y Ferraro (2003); Foio y Espínola (2004); García de Fanelli (2004); Porto y Di Gresia (2004) y Di Gresia (2007), y los que responden a una mirada más psicosocial, entre los que se destacan - por su profundidad a partir de un análisis longitudinal-los trabajos de Aparicio (2008).

García de Fanelli (2006) sostiene que el bajo nivel de selectividad y la enseñanza gratuita predominantes en las universidades públicas del país no son condiciones suficientes para garantizar la permanencia de los sectores de menor ingreso en la educación superior. Consecuentemente, a partir de indicadores elaborados 
por el Sistema de Información de Tendencias Educativas en América Latina (Siteal), ${ }^{2}$ dicha autora describe algunos rasgos sociodemográficos y laborales de aquellos que abandonaron la educación superior. García de Fanelli señala que los dos principales factores que inciden en la deserción estudiantil son el estado civil -teniendo más probabilidades de terminar los estudios aquellos que no son jefes o cónyuges- y la necesidad de ingresar al mercado laboral reflejada en la mayor tasa de actividad de los que abandonan los estudios respecto de aquellos que continúan estudiando. Además, teniendo en cuenta las investigaciones realizadas en los últimos años sobre el mercado de trabajo de la Argentina, la autora concluye que no sólo la tasa desocupación es un $50 \%$ más baja entre los graduados del nivel superior respecto de los egresados del nivel medio, sino que también los graduados del nivel superior están ocupados en un mayor porcentaje en el sector formal y obtienen mejores condiciones laborales en general. De ello se desprende que el fenómeno de la deserción en el nivel superior es un problema social cuya solución reviste una importancia estratégica, tanto para elevar el capital humano de la población económicamente activa, como para mejorar las condiciones particulares de vida de los jóvenes, especialmente las de aquellos de menor nivel socioeconómico (García de Fanelli, 2006, p. 15).

En su estudio, Beguet et al. (2001) buscan identificar en el primer año de estudios las variables sociodemográficas, los antecedentes escolares, la autopercepción de razones de ingreso y la permanencia en los estudios, las expectativas de inserción profesional y su relación con el rendimiento académico. Los resultados señalaron que tanto las características sociodemográficas de los estudiantes, como las demás variables incluidas en el estudio se mantuvieron sin cambios. En cuanto al rendimiento académico exitoso en el primer año, se constató que este está relacionado con las razones de elección de la carrera y la continuidad en los estudios. Para los demás factores no se encontró relación con el rendimiento. En la Universidad Nacional de Tres de Febrero, Lorenzano y Ferraro (2003) analizaron el perfil del estudiante y su rendimiento académico. Para ello, describieron las características sociodemográficas de los estudiantes y las relacionaron con los resultados

Pertenece al Instituto Internacional de Planeamiento Educativo (IIPE-Unesco Buenos Aires) y de la Organización de Estados Iberoamericanos (OEI). Ha confeccionado una serie de indicadores sobre la base de la Encuesta Permanente de Hogares con el fin de analizar las cuestiones del acceso, la permanencia y el perfil social de los graduados de la educación superior comparados con los egresados de la educación media. obtenidos en los tres primeros meses de su carrera. Como conclusiones, destacaron que factores como la edad de los estudiantes, el lapso de tiempo desde que egresaron de la secundaria, el género, la situación laboral y el turno elegido, influyen en el rendimiento académico y la retención. Por el contario, en este trabajo no se encontró relación entre el rendimiento académico y la retención con factores tales como el tipo de gestión de la escuela de origen (pública o privada), el nivel educativo de los padres y el estado civil.

Solimano (1997), en su tesis sobre el ingreso de los estudiantes y su vinculación con el rendimiento, concluye que los estudiantes que ingresan en mejores condiciones académicas, medido en función del curso de ingreso, obtienen un mejor rendimiento en los exámenes parciales y finales de las materias básicas del primero año de la universidad. Además, halló que los estudiantes que cumplen con los requisitos de los exámenes parciales aprueban con mayor facilidad los exámenes finales, y que es menor el rendimiento académico de los estudiantes que trabajan.

Los resultados de un estudio empírico de Spady (1970) indican que, en un periodo de cuatro años, el rendimiento académico formal fue el factor que mejor explicaba la deserción estudiantil en ambos sexos. En relación con estos hallazgos, el Departamento de Economía de la Universidad Nacional de la Plata aplicó una encuesta a 4.676 estudiantes de la Facultad de Economía para analizar la relación entre el rendimiento académico y sus determinantes (Porto y Di Gresia, 2004). De acuerdo con este estudio, resulta que son variables explicativas significativas del rendimiento estudiantil: la carrera que cursa el estudiante, el género, la edad, el nivel educativo de los padres, la cantidad de horas que trabaja y las regulaciones de la Facultad en cuanto a la condición de alumno regular. Por el contrario, resultó que tanto el tipo de escuela secundaria (pública o privada) como el lugar de procedencia del estudiante no tienen influencia sobre el rendimiento académico. Asimismo, otro estudio muestra que el desempeño académico de los estudiantes es superior para las mujeres, para los estudiantes que ingresan más jóvenes a la universidad, para quienes provienen de hogares con padres más educados y para los estudiantes que no trabajan (Di Gresia, 2007). Otros estudios llevados a cabo en este Departamento de Economía tuvieron como objetivo analizar la relación entre el rendimiento académico y los mecanismos de admisión a la universidad. Porto, Di Gresia y López-Armengol (2004) encontraron que los resultados de las pruebas de eva- 
luación diagnóstica no eran una variable significativa para explicar el rendimiento académico, tanto en el primer ciclo como en el segundo. En su estudio sobre la relación entre las aptitudes educativas - medidas por el Test de Aptitudes Diferenciales (DAT) - el rendimiento académico y la deserción, Corengia, Pita, Mesurado y Centeno (2013) encontraron que si bien las aptitudes educativas puede predecir el rendimiento en el primer año de la carrera, existe una relación "moderada a baja" entre estas aptitudes y la tasa de deserción.

El centro de interés de los trabajos de Aparicio (2008) redunda en dilucidar las raíces psicosociales del fracaso en la educación superior. Desde su perspectiva de estudio, el logro o el fracaso se ofrecen como un problema que conjuga tres dimensiones: a) condicionantes personales, psicosociales y socioculturales; b) factores pedagógico-institucionales y c) factores estructurales como el mercado de trabajo. A partir de sus estudios, Aparicio (2008) concluye que la aproximación a la comprensión del fenómeno exige recuperar al sujeto y su estructura, el plano micro y el plano contextual. El éxito o el fracaso no se explican sólo por la crisis que toca a la universidad; tampoco por las condiciones que signan el mercado de empleo, sometido al cambio más vertiginoso que se haya conocido; antes bien, confluyen bajo él imbricándose de un modo complejo, aspectos de base, vivenciales e institucionales: ningún factor solo basta para explicar una conducta (p. 22).

En el nivel internacional, la deserción viene recibiendo una atención creciente, alineada con los objetivos de los gobiernos de incrementar significativamente la población que accede a la educación superior y completa su formación en ella. Por lo tanto, se puede decir que se está dando un giro desde el estudio de la deserción hacia la retención, y más específicamente a estudiar los factores - tanto institucionales como los provenientes de las características de los estudiantesque su contribuyen al éxito (Habley, Bloom y Robbins, 2012). De este modo, el foco de las causas de abandono se ponen tanto en el estudiante como en la institución. Según Hossler, Ziskin, Moore III y Wakhungu (2008), las prácticas institucionales también son llamadas por algunos autores más recientes como policy levers (palancas de políticas) cuyo rol en relación con la retención estudiantil debe ser aún estudiado (Braxton y McClendon, 2001-2002; Pascarella y Terenzini, 1991). Asimismo, dichos autores sostienen que para poder indagar sobre el rol de estas "palancas", primero es necesario indagar cómo las prácticas institucionales afectan la retención estudiantil, especialmente por medio de estudios que incluyan múltiples instituciones. Si bien no se puede negar que las experiencias de los estudiantes en la universidad son moldeadas por las políticas y prácticas diarias que se desarrollan en el campus, las instituciones carecen de la evidencia para conocer de qué manera las políticas y prácticas institucionales afectan la retención estudiantil. Por último, los autores concluyen que la habilidad para moldear la retención estudiantil en su contexto institucional, e identificar programas y políticas que promuevan la retención dentro de ese contexto, son dos aspectos que las instituciones deben tener en cuenta con miras a lograr el éxito de sus estudiantes.

El concepto de éxito de los estudiantes es entendido en términos de finalización de los estudios de grado y/o de alcanzar las metas educativas propuestas. Como prueba de ello, se puede decir que las más recientes e influyentes publicaciones en este campo incluyen el término éxito de los estudiantes en sus títulos, así: Student Success in College (Kuh, Kinzie, Schuh, Whitt y Associates, 2005), Fostering Student Success in the Campus Environment (Kramer y Associates, 2007) y College Student Retention: Formula for Student Success (Seidman, 2005). Más aún, Vincent Tinto, uno de los primeros autores que ha investigado y escrito sobre la deserción, prueba este cambio de enfoque hacia la retención a través de los títulos de sus obras a medida que pasa el tiempo. En 1993 publicó su obra Leaving College: Rethinking the Causes and Cures of Student Attrition, y en el 2012 su obra publicada se titula: Completing College: Rethinking Institutional Action. A partir del título de esta obra, se puede vislumbrar cómo la investigación se desplaza del estudio de la deserción para centrarse más en el fenómeno de la retención, y más específicamente en los factores institucionales. En su última obra, Tinto sostiene que la retención no es la imagen opuesta a la deserción, dado que si bien ambos procesos están necesariamente relacionados, no basta con entender las razones por las cuales los estudiantes desertan para poder ayudarles a permanecer en sus estudios y en la universidad.

A partir de la bibliografía consultada, se puede afirmar que el concepto de éxito de los estudiantes abarca nuevos términos como involucramiento (involvement), integración (integration) y compromiso (engagement), pertenecientes a distintas perspectivas teóricas que desde mediados de los años setenta han sido propuestas y desarrolladas por distintos autores. Astin (1975) fue el primero en articular el concepto de involucramiento en su libro Preventing Students from Dropping Out. Allí, 
define el involucramiento como la cantidad de energía física y psicológica que un estudiante invierte en su experiencia académica. La premisa básica del involucramiento según este autor es que los estudiantes aprenden más cuanto más involucrados están en los aspectos académicos y sociales de su experiencia universitaria. Esto quiere decir que una mayor participación de los alumnos en experiencias significativas en el campus contribuye a un mayor aprendizaje y desarrollo personal. Consecuentemente, Astin postula que la institución puede contribuir a un mayor involucramiento del estudiante por medio del ofrecimiento de experiencias educativas enriquecedoras tanto dentro como fuera del aula.

Más tarde, Tinto (1993) introdujo los conceptos de integración académica y social, poniendo el énfasis en los factores institucionales como explicativos del abandono estudiantil. Su teoría de la integración, basada en el modelo sociológico de Spady (1970), postula que el abandono estudiantil es el resultado tanto del nivel en que los estudiantes comparten las actitudes y creencias de sus compañeros y profesores, como del grado en que adhieren a las reglas y los requerimientos de la institución (cultura institucional). Luego, en su libro más reciente Completing College: Rethinking Institutional Action (2012), Tinto sostiene que la investigación sobre retención converge en cuatro factores que la favorecen: expectativas, apoyo, evaluación y feedback e involucramiento y compromiso. Dentro del factor de apoyo distingue entre apoyo académico, social y financiero. Estos mismos factores son los que Seidman (2005) publica en su obra: College Student Retention: Formula for Student Success para explicar la retención en la educación superior. Para Tinto, entonces, los estudiantes tienen más probabilidades de tener éxito cuando: a) se generan altas expectativas hacia ellos, b) se les provee de apoyo social y académico, c) se evalúa su desempeño y provee feedback frecuentemente, y d) se los involucra activamente con otros en la universidad, especialmente en el aula. Además, como bien sostiene Tinto (1993), en su libro Leaving College: Rethinking the Causes and the $\mathrm{Cu}$ res of Student Attrition, en los primeros años, lo más importante para el estudiante, en pos de su permanencia en la institución, es el grado en que se integra socialmente a la universidad más que los aspectos académicos de la experiencia estudiantil.

Por último, Kuh (2001) avanza sobre el trabajo de Astin y formula el concepto de student engagement (compromiso estudiantil). Como bien sostiene Coates (2005), la esencia de este concepto reside en conocer cuán involucrados están los estudiantes universitarios en diversas actividades educativas facilitadoras de un aprendizaje de calidad. Consecuentemente define al student engagement como un amplio constructo que abarca variables académicas y no académicas de la experiencia estudiantil, que comprenden los siguientes aspectos:

- Nivel de desafío académico (Level of academic challenge): hace referencia a la participación de los alumnos en actividades académicas desafiantes que impliquen altas expectativas del logro académico, tales como método intensivo de lectura, escritura y preparación para las clases, rigurosas experiencias para el fin de las carreras como tesis, tesinas, etcétera.

- Participación en métodos de aprendizaje activo y colaborativo (active and collaborative learning): hace referencia al aprendizaje de un colega, a las comunidades de aprendizaje, al aprendizaje en la comunidad local y servicio, entre otros.

- Interacciones con el profesorado dentro y fuera del aula (formative communication with academic staff).

- Participación en experiencias educativas enriquecedoras (involvement in enriching educational experiences) tales como la utilización apropiada de las tecnologías que facilitan el aprendizaje y promueven la colaboración entre estudiantes y profesores, la infusión de experiencias diversas, estudiar en el extranjero, servicio en la comunidad, pasantías y prácticas profesionales, etcétera.

- Clima de tolerancia y apoyo (supportive learning environment o supportive campus environment): hace referencia a los programas de transición que dan la bienvenida y afirman a los estudiantes nuevos, redes de consejeros (tutores) que responden a las necesidades académicas y no académicas de los estudiantes, apoyo de los colegas, múltiples redes de seguridad o sistema de alerta para alumnos en riesgo (safety nets) y residencias.

Otros autores como Pascarella y Terenzini (2005) sostienen que las actividades extracurriculares, junto con otros factores como el clima de interacción entre estudiantes, facilitan la obtención de un título o maximizan el logro y la persistencia académica. No obstante, estos autores enfatizan en que el factor determinante del impacto de la universidad en la permanencia o abandono es el compromiso o esfuerzo individual del alumno, por lo que es importante desde la gestión educativa ver cómo se puede modificar y repercutir en ello para fomentar ese compromiso inicial. 


\section{Perspectivas teóricas sustantivas y estudios empíricos sobre deserción}

Las perspectivas teóricas sobre retención pueden distinguirse en distintos modelos conceptuales según se basen en teorías sociológicas, psicológicas, organizacionales, económicas o culturales. Todas ellas, a pesar de su complejidad, no pueden explicar por sí solas el fenómeno de la retención. Además, es importante señalar aquí que existen numerosos estudios que intentan medir el impacto de algunas intervenciones institucionales que fueron implementadas con el fin de aumentar las tasas de retención. Las perspectivas sociológicas son las que dominaron el estudio de la retención en los últimos cuarenta años (Habley et al., 2012). Estas perspectivas hacen referencia a la influencia de las estructuras y fuerzas sociales en las decisiones de abandono de los estudiantes (Seidman, 2005).

Spady (1970) fue el primero en proponer un modelo globalmente reconocido para explicar la deserción estudiantil en el nivel educativo superior. Dicho modelo, basado en el que utilizó Durkheim (1951) para explicar el suicidio, suponía la existencia de cinco variables independientes que inciden directamente en la integración social de los estudiantes; estas son: potencial académico del estudiante; congruencia normativa; rendimiento académico; desarrollo intelectual y apoyo social de los compañeros. Además, estas cinco variables influían indirectamente en la variable "decisión de abandonar" por medio de dos nuevas variables intervinientes: satisfacción y compromiso institucional. Unos años más tarde, Tinto (1975) creó -basándose en el modelo multivariado de Spady- el modelo interaccionista cuyo foco principal está en la integración académica y social. Para dicho autor, cuanto mayor es el nivel de integración social y académica del estudiante, mayor será su nivel de compromiso con la institución y su objetivo de terminar los estudios. El abandono de los estudios consiste en un proceso longitudinal que ocurre debido a que el sujeto le otorga significado a sus interacciones con las dimensiones formales e informales de una determinada institución educativa (Braxton, Sullivan y Johnson, 1997; Tinto, 1986 y 1993). En sus investigaciones posteriores, Tinto (1993) postuló que grupos de estudiantes diferentes, como por ejemplo adultos y transferidos, requieren políticas y programas específicos de retención por cada grupo, así como distintos tipos de instituciones (urbanas, públicas grandes, no residenciales, etcétera) también requieren diversos tipos de programas y políticas de retención.

Respecto de las perspectivas psicológicas, Bean y Eaton (2002) formularon un modelo basado en cuatro procesos psicológicos que contribuyen a la integración académica y social; estos son: autoeficacia positiva, manejo del estrés, aumento de la eficacia y locus de control interno. Estos autores postulan que las características de los estudiantes al entrar a la institución, como por ejemplo el comportamiento anterior y las creencias, moldean la percepción que tienen los estudiantes del ambiente-entorno de la universidad. Luego, las interacciones con el entorno institucional también redundan en procesos psicológicos que inciden en la motivación de los estudiantes. Estos procesos sufren ajustes y cambios permanentes tanto por la interacción con el entorno institucional y externo, como por las influencias recibidas por parte del cónyuge (o pareja) y los padres. Por su parte, a partir de su teoría del involucramiento, Astin (1985) sostiene que los estudiantes aprenden más y mejor cuanto más involucrados están en los aspectos académicos y sociales de su experiencia universitaria. Como ya se mencionó anteriormente, define al involucramiento como la cantidad de energía física y psicológica que un estudiante invierte en su experiencia académica. En un estudio empírico que utiliza información proveniente de una encuesta anual a los estudiantes de primer año, realizada en la Universidad de California por el Instituto de Investigación de Educación Superior (HERI, por sus siglas en inglés), Astin encontró que las tres maneras más relevantes de involucramiento eran el académico, con los profesores y con el grupo de pares.

En cuanto a las perspectivas organizacionales, se hace referencia al modelo creado por Bean (1980 y 1983) para explicar el abandono universitario, el cual es una adaptación del de Price y Mueller (1981) sobre la rotación de los empleados en la organización del trabajo. En este modelo existen diez variables exógenas que repercuten sobre la variable satisfacción, la que a su vez influye en la intención de un estudiante de abandonar sus estudios. De las diez variables, cinco son las mismas que en el modelo de Price y Mueller: participación, rutinización, comunicación instrumental, integración y justicia distributiva, y las otras cinco fueron agregadas por Bean: calificaciones, valor práctico, desarrollo, asignaturas y pertenencia a organizaciones del campus. Todas las variables tienen un efecto positivo en la satisfacción, a excepción de la rutinización. Además, Bean postula que existen otras dos variables que tienen 
influencia sobre la intención de un estudiante de abandonar sus estudios, una es la condición de estar casado, y la otra la define como oportunidad. Esta última implica la oportunidad que tienen los estudiantes de poder transferirse a otra institución de educación superior.

Basada en la teoría del capital humano (Becker, 1964), la perspectiva económica sostiene que los estudiantes analizan los costos y los beneficios de continuar sus estudios en una institución. En consecuencia, desde esta perspectiva, el abandono se produce cuando los costos que percibe un estudiante por asistir a una institución en particular exceden los beneficios de graduarse en ella. A pesar de que las cuestiones monetarias son costos directos (matrícula, cuotas, ingresos no percibidos), la perspectiva económica también se aplica a los costos indirectos, como son el tiempo y la energía que los estudiantes tienen que invertir en cualquier actividad así como a la institución en sí misma. Otros autores como St. John, Cabrera, Nora y Asker (2000) también analizan exhaustivamente las influencias de lo económico en la retención de los estudiantes.

El interés y con ello los estudios sobre el impacto de los factores culturales en la persistencia estudiantil fueron ganando importancia a medida que creció la diversidad de los ingresantes a las universidades. La perspectiva cultural se centra no sólo en los estudiantes pertenecientes a las minorías raciales o étnicas, sino también en los estudiantes internacionales y en los que son primera generación de estudiantes en sus familias.

Cabe señalar que cada una de estas perspectivas es de utilidad para explicar algunos elementos que contribuyen a comprender la retención, pero ninguna puede comprehender y abarcar todos los factores que la influyen. La decisión de un individuo de permanecer o abandonar los estudios no está basada en una de estas perspectivas, sino más bien en una mezcla de ellas, así como en creencias, actitudes y circunstancias adicionales e idiosincráticas. Consecuentemente, lo que realmente desafía a los investigadores y a las personas que toman decisiones en la práctica es la integración de estas perspectivas en un modelo integral (Habley et al., 2012).

Por un lado, Pascarella (1985) desarrolló un modelo causal para poder explicar el patrón de influencias involucradas en el impacto de la educación superior en el aprendizaje y desarrollo cognitivo de los sujetos. Para este autor, la calidad del esfuerzo del estudiante, sus características previas y las interacciones con agentes de socialización tienen un impacto en su aprendizaje y desarrollo cognitivo. Los resultados de su estudio empírico indicaron que las facilidades que brinda una institución de poder vivir allí además de estudiar (instalaciones residenciales) y el principal grupo de pares influenciaban fuertemente el rendimiento académico. Un factor que también demostró tener una importante relación, aunque en menor grado, fue la interacción informal entre estudiantes y profesores. Asimismo, los principales hallazgos de una investigación cuyo objetivo central fue analizar los programas de retención estudiantil de pregrado de las instituciones de educación superior colombianas, ponen de relieve la importancia del rol "más humanizador" del docente como actor que acompaña al estudiante. También se destaca la necesidad de que el profesor se involucre más activamente en las investigaciones sobre deserción y su idoneidad para motivar al individuo hacia el aprendizaje (Pineda-Báez, Pedraza-Ortiz y Moreno, 2011).

Por otro lado, Castaño et al. (2004) también analizan el problema de la deserción estudiantil integrando variables individuales, académicas, socioeconómicas e institucionales, con el fin de obtener conclusiones más contundentes sobre este complejo fenómeno. De esta manera, se diferencian de estudios anteriores sobre la deserción universitaria, los cuales han tratado de explicarla, con poco "éxito", sólo a partir de alguno de los factores que teóricamente han sido propuestos.

En el marco de estos estudios empíricos, en la siguiente sección se presentan las principales acciones y programas llevados a cabo a lo largo de los últimos cuarenta años para promover la retención en la educación superior.

\section{Avances hacia programas de retención en las universidades}

En la práctica se han llevado a cabo numerosas actividades y programas con el fin de promover la retención en las universidades. Los estudios sobre estas prácticas son los que han nutrido las investigaciones sobre el tema de la retención. La mayoría de estos se pueden encontrar en países como los Estados Unidos, Australia, algunos países de Europa y, en menor medida, en países de Latinoamérica. Todos se focalizan en el primer año de la carrera, dado que la mayor deserción se da en el paso del primero al segundo año (Ezcurra, 2005 y 2007; Robinson, 1990). Más aún, las acciones para aumentar la retención empiezan desde que el alumno entra en el proceso de admisión, es decir, desde el primer contacto que hace el estudiante con la institución. Los programas de orientación y las evaluaciones 
previas a la entrada son algunas de las acciones que se han llevado a cabo en la mayoría de las instituciones.

Luego, ya empezado el primer año de la carrera, existe una gran diversidad de programas a implementar. Ejemplo de ello son los programas de asistencia en la transición (transition assistance programs) que se focalizan en ayudar a los estudiantes a adaptarse a la universidad tanto en los aspectos académicos como en los sociales. También están los programas de contacto temprano con las comunidades (early contact/community programs) cuyo fin es promover la participación de los nuevos estudiantes con los miembros de las comunidades sociales y académicas de la universidad. Además, existe la posibilidad de que los ingresantes formen grupos con base en sus intereses intelectuales y académicos y luego por grupos se inscriban en las mismas materias y seminarios durante el primer cuatrimestre. Los sistemas de alerta para alumnos en riesgo (safety nets) es otra práctica institucional que se ha implementado en las universidades con el fin de alertar tempranamente al estudiante de un bajo rendimiento académico que puede poner en peligro su permanencia en la institución y ayudarlo a regularizar su situación. Otras acciones que ponen en práctica las universidades son los programas de asesoramiento obligatorios y los programas integrales para el primer año (integrated first year programs). Es importante mencionar también los programas de ayuda financiera, o las becas que permiten a los estudiantes costear sus estudios con el fin de que no deserten por un tema meramente económico (Tinto, 1993).

En la mayoría de las universidades de los Estados Unidos existe un gran movimiento que se denomina First Year Experience (FYE) que consiste en la combinación de todos los servicios, los programas, las actividades curriculares y extracurriculares que promueven la transición de un estudiante desde la secundaria hasta la universidad y proporcionan las bases para el éxito académico en una institución determinada. El FYE comienza en el momento de la admisión y continúa hasta la finalización del primer año en la universidad. Su oferta académica es altamente variada, aunque la mayoría de los programas generalmente incluyen la enseñanza de habilidades para la gestión del tiempo, estrategias para la lectura de textos, estrategias para tomar exámenes, técnicas de redacción de documentos, la exploración de valores (incluso considerando el porqué uno elige ir a la universidad), tomar notas efectivas y la alfabetización tecnológica e informacional. Además, los cursos o programas pueden abarcar la enseñanza de "habilidades blandas" que se relacionan con el éxito profesional y personal en la vida, incluyendo la toma de decisiones, los problemas de relaciones y diversidad, la salud, el estrés y, cada vez más, la alfabetización en habilidades financieras (Feldman y Zimbler, 2011).

En Colombia, los principales programas de retención llevados adelante por las universidades son las monitorías, el apoyo de pares, las consejerías y los cursos de matemáticas, ciencias básicas y lecto-escritura, los cursos de inducción, la capacitación permanente de docentes y las consultas médicas, odontológicas, de nutrición y psicológicas. Además, también cuentan con una variedad de programas de ayuda económica ( $\mathrm{Pi}$ neda-Báez et al., 2010). Los resultados de un estudio de carácter cualitativo, cuyo objetivo principal fue describir e interpretar las vivencias de los estudiantes en algunos programas enmarcados en una política de retención en la Universidad de La Sabana, muestran que los sujetos recurren a una gran variedad de programas con miras de robustecer su intención de continuar vinculados a la universidad. Estos programas no sólo fortalecen la integración académica sino también el tejido social del estudiante dentro de la institución (PinedaBáez y Pedraza-Ortiz, 2009).

\section{Conclusiones}

La exhaustiva revisión bibliográfica da cuenta de que existe una relación importante entre el aprendizaje y la persistencia producto de la interacción entre el involucramiento y la calidad del esfuerzo del estudiante. Consecuentemente, se puede afirmar que los alumnos no son los únicos responsables de su propio esfuerzo, sino que también influye lo que al respecto realizan las instituciones con el fin de involucrarlos (student engagement) con ella y sus miembros: profesores, administrativos, directivos y pares. Especial relevancia tienen las acciones y los programas que se focalizan en el aprendizaje dentro del aula, más específicamente en el contacto entre profesores y alumnos. El objetivo de lograr una mayor retención es sólo el vehículo para conseguir el objetivo primordial de la educación superior: el aprendizaje de los estudiantes y su éxito en la finalización de sus estudios.

Además, la presente discusión bibliográfica muestra cómo la investigación sobre esta temática se fue desplazando desde el fenómeno de la deserción hacia el de la retención, haciendo un fuerte hincapié en los programas que tuvieron éxito en asegurar la permanencia de los estudiantes en la universidad. En consistencia 
con el modelo de retención planteado por Tinto (1993 y 2012), dichos programas resultan exitosos cuando fortalecen la integración académica y social del estudiante dentro de la institución.

A partir de las teorías conceptuales y los estudios empíricos relevados, se aportaron elementos que permiten pensar desde diversos enfoques y contextos un modelo integrador que ayude a comprender el complejo y multidimensional fenómeno de la deserción, para luego ponerlo en práctica y aumentar así las tasas de retención. Sin duda, es necesario continuar investigando esta problemática social - y no sólo educativa- desde perspectivas cualitativas y cuantitativas que recuperen al sujeto y su estructura tanto en el plano micro como en el plano contextual.

\section{Referencias}

Aparicio, M. (2005). Les facteurs psychosociaux en relation avec la réussite universitaire et professionnelle. (Tesis de Doctorado en Ciencias de la Educación). Universidad René Descartes, Paris V, Sorbonne, Francia.

Aparicio, M. (2008). La deserción universitaria y su relación con factores motivacionales. Diálogos Pedagógicos, 6(11), 11-26.

Astin, A. W. (1975). Preventing Students from Dropping Out. San Francisco: Jossey-Bass.

Astin, A. W. (1985). Achieving Educational Excellence: A Critical Assessment of Priorities and Practices in Higher Education. San Francisco: Jossey-Bass.

Bean, J. P. (1980). Student attrition, intensions and confidence. Research in Higher Education, 17, 291-320.

Bean, J. P. (1983). The Application of a Model of Turnover in Work Organizations to the Student Attrition Process. The Review of Higher Education, 6, 127-148.

Bean, J. y Eaton, S. B. (2002). The Psychology Underlying Successful Retention Practices. J. College Student Retention, 3(1), 73-89.

Becker, G. S. (1964). Human Capital. New York: National Bureau of Economic Research.

Beguet, B., Kohan, N., Castro-Solano, A. y Renault, G. (2001). Factores que intervienen en el rendimiento académico de los estudiantes de psicología y psicopedagogía. Revista Científica de la Dirección de Evaluación y Acreditación de la Secretaría General de la Universidad del Salvador-USAL, 1(1), 1-18. Recuperado de http://www. salvador.edu.ar/uc4-pub-01-1-1-04.htm.

Bellei, C. (2003). ¿Ha tenido impacto la Reforma Educativa en Chile? En C. Cox (Ed.), Políticas educacionales en el cambio de siglo (pp. 125-209). Ministerio de Educación de Chile: Editorial Universitaria.

Berger, J. B. (2002). Understanding the organizational nature of student persistence: Empirically-based recommendations for practice. Journal of College Student Retention, 3(1), 3-21.

Berger, J. B. y Milem, J. F. (1999). The Role of Student Envolvement and Perceptions of Integration in a Causal Model of Student Persistence. Research in Higher Education, 40(6), 641-664.

Braxton, J. M. y McClendon, S. A. (2001-2002). The Fostering of Social Integration through Institutional Practice. Journal of College Student Retention: Research, Theory and Practice, 3(1), 57-71.

Braxton, J. M., Sullivan, A. S. y Johnson, R. M. (1997). Appraising Tinto's Theory of College Student Departure. Higher Education: A Handbook of Theory and Research, 12, 107-164

Braxton, J. M. y Mundy, M. E. (2002). Powerful Institutional Levers to Reduce College Student Departure. Journal of College Student Retention, 3(1), 91-118.

Castaño, E., Gallón, S., Gómez, K. y Vásquez, J. (2004). Deserción estudiantil universitaria: una aplicación de modelos de duración. Lecturas de Economía, 60, 41-65.

Centro de Estudios de la Educación Argentina (CEA). (2012). Necesitamos más graduados. Recuperado de http://www. ub.edu.ar/centros_de_estudio/cea/cea_numero_5.pdf

Coates, H. (2005). The Value of Student Enagagement for Higher Education Quality Assurance. Quality in Higher Education, 11(1), 25-36.

Corengia, A., Pita, M., Mesurado, B. y Centeno, A. (2013). La predicción de rendimiento académico y deserción en estudiantes universitarios. Revista Liberabit, 19(1), 101-112.

Di Gresia, L. (2007). Rendimiento académico universitario. Documento de la Universidad Nacional de la Plata. Recuperado de http://www.aaep.org.ar/anales/works/ works2007/digresia.pdf

Durkheim, E. (1951). Suicide. New York: Free Press.

Ezcurra, A. M. (2005). Diagnóstico preliminar de las dificultades de los alumnos de primer ingreso a la Educación Superior. Perfiles Educativos, 37(107), 118-133.

Ezcurra, A. M. (2007) Los estudiantes de nuevo ingreso: democratización y responsabilidad de las instituciones universitarias. San Pablo: Universidade de Sao Paulo Pro-reitoria de Graduacao.

Feldman, R. S. y Zimbler, M. (2011). Engendering College Student Success: Improving the First Year and Beyond. Massachusetts: The McGraw-Hill Research Foundation.

Foio, M. y Espínola, A. (2004). Seguimiento académico de los ingresantes 2002. Documentos de la Secretaría de Planificación de la Universidad Nacional del Nordeste. Recu- 
perado de http://eluniversitario.unne.edu.ar/2004/44/ pagina/Informe\%20Completo.pdf

García de Fanelli, A. M. (2004). Indicadores y estrategias en relación con el abandono y la graduación universitarios. En C. Marquis (Ed.), La Agenda Universitaria. Buenos Aires: Colección Educación Superior, Universidad de Palermo.

García de Fanelli, A. M. (2006). Acceso, abandono y graduación en la educación superior argentina. Debate 5. Siteal. Buenos Aires: IIPE - Unesco, OEI.

García de Fanelli, A. M. y Trombetta, A. (1996). Diferenciación institucional y reformas curriculares en los sistemas de educación superior. Buenos Aires: Ministerio de Cultura y Educación.

Gekowski, N. y Schwartz, S. (1961). Student mortality and related factors. Journal of Educational Research, 54, 192-194.

Habley, W., Bloom, J. y Robbins, S. (2012). Increasing Persistence. Research Based Strategies for College Student Success. San Francisco, CA: Jossey Bass.

Hossler, D., Ziskin, M., Moore III, J. V. y Wakhungu, P. K. (2008). The role of institutional practices in college student persistence. Results from a policy-oriented pilot study. Annual Forum of the Association for Institutional Research (AIR). WA, Seattle.

Iffert, R. E. (1957). Retention and withdrawal of college students. U.S. Office of Education, Bulletin 1957, 1. Washington, D. C.: U.S. Government Printing Office.

Kramer, G. L. y Associates (2007). Fostering Student Success in the Campus Community. San Francisco, CA: Jossey-Bass.

Kuh, G. D. (2001). Assessing what really matters to student learning: Inside the National Survey of Student Engagement. Change, 33(3), 10-17.

Kuh, G; Kinzie, J; Schuh, J; Whitt, E. y Associates (2005). Students Success in College : Creating Conditions that Matter. San Francisco, CA: Jossey Bass.

Lorenzano, C. y Ferraro, M. (2003). Perfil de los ingresantes de la Universidad Nacional de Tres de Febrero: Rendimiento y posibles causas del fracaso académico. Artículo presentado en el II Congreso Nacional de Investigación Educativa, México.

McNeely, J. H. (1937). College student mortality. U.S. Office of Education, Bulletin 1937, 11. Washington, DC: U. S. Government Printing Office.

Panaia, M. (Coord.). (2013). Abandonar la Universidad con o sin título. Buenos Aires-Madrid: Miño y Dávila.

Panos, R. J. y Astin, A. W. (1967). Attrition among College Students. ACE Research Reports, 2(4). Washington, D. C.: Office of Research, American Council on Education.
Pantages, T. J. y Creedon, C. F. (1978). Studies of college attrition: 1950-1975. Review of Educational Research, 48(1), 49-101.

Pascarella, E. T. (1985). Racial Differences in Factors Associated with Bachelor's Degree Completion: A Nine Year Follow Up. Research in Higher Education, 23(4), 351-373.

Pascarella, E. T. y Terenzini, P. T. (1991). How College Affects Students. Vol. 1: Findings and Insights from Twenty Years of Research. San Francisco: Jossey-Bass.

Pascarella, E.T. y Terenzini, P.T. (2005). How College Affects Students: Vol. 2. A Third Decade of Research. San Francisco, CA: Jossey- Bass.

Pineda-Báez, C., Pedraza-Ortiz, A., Baquero, M., Dussán, F. y Ramírez, M. (2010). La voz del estudiante: El éxito de programas de retención universitaria. Bogotá: Unesco IESALC, Universidad de La Sabana.

Pineda-Báez, C.; Pedraza-Ortiz, A. y Moreno, I. (2011). Efectividad de las estrategias de retención universitaria: La función del docente. Educ. Educ., 14(1), 119-135.

Pineda-Báez, C. y Pedraza-Ortiz, A. (2009). Programas exitosos de retención estudiantil universitaria: Las vivencias de los estudiantes. Revista Virtual Universidad Católica del Norte, 28, 1-30. Recuperado de http://revistavirtual. ucn.edu.co/

Porto, A. y Di Gresia, L. (2004). Rendimiento de estudiantes universitarios y sus determinantes. Revista de Economía y Estadística, XLII, 94-113.

Porto, A., Di Gresia, L. y López-Armengol, M. (2004). Admisión a la universidad y rendimiento de los estudiantes. Artículo presentado en la xxxix Reunión Anual de la AAEP, Universidad Católica Argentina.

Price, J. L. y Mueller C. W. (1981). A Causal Model of Turnover for Nurses. The Academy of Management Journal, 24(3), 543-565.

Robinson, R. (1990). Understanding the Gap between Entry and Exit: A Cohort Analysis of African American Students Persistence. Journal of Negro Education, 59, 207-218.

Seidman, A., (Ed) (2005). College Student Retention: Formula for Student Success. Westport, CT: ACE/Praeger.

Sexton, V. S. (1965). Factors contributing to attrition in college populations: Twenty five years of research. Journal of General Psychology, 72, 301-326.

Solimano, A. (1997). Un estudio sobre la problemática del ingreso y su vinculación con el rendimiento (Tesis de grado). Biblioteca de la Facultad de Ciencias Económicas y Estadísticas UNR.

Spady, W. (1970). Dropout from Higher Education: An Interdisciplinary Review and Synthesis. Interchange, 1, 64-85. 
St. John, E. P., Cabrera, A. F., Nora, A. y Asker, E. H. (2000) Economic Influences on Persistence Reconsidered: How Can Finance Research Inform the Reconceptualization of Persistence Models? En J. M. Braxton (Ed.), Reworking the Student Departure Puzzle (pp. 29-47). Nashville: Vanderbilt University Press.

Summerskill, J. (1962). The American College (pp. 627-657). New York: Wiley.

Tinto, V. (1975). Dropouts from Higher Education: A Theoretical Synthesis of Recent Research. Review of Educational Research, 45, 89-125.
Tinto, V. (1986). Theories of Student Departure Revisited. Higher Education: A Handbook of Theory and Research, 2, 359-384.

Tinto, V. (1990). Principles of Effective Retention. Journal of the Freshman Year Experience, 2(1), 35-48.

Tinto, V. (1993). Leaving College. Rethinking the Causes and Cures of Student Attrition. Chicago: University of Chicago Press.

Tinto, V. (2012). Completing College. Rethinking Institutional Action. Chicago: University of Chicago Press. 